\title{
Research Paper: \\ Effect of Group Play Therapy on Emotional/Behavioral Problems of Chil- dren With Attention Deficit Hyperactivity Disorder Aged 6-12 Years
}

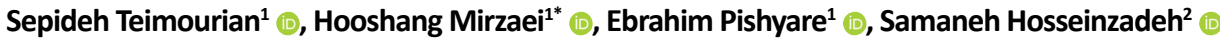

1. Department of Occupational Therapy, School of Rehabilitation Sciences, University of Social Welfare and Rehabilitation Sciences, Tehran, Iran 2. Department of Biostatistics, School of Rehabilitation Sciences, University of Social Welfare and Rehabilitation Sciences, Tehran, Iran.

\begin{tabular}{|c|c|}
\hline $\begin{array}{l}\text { Use your device to scan } \\
\text { and read the article online }\end{array}$ & Cltation Teimourian S, Mirzaei H. Pishyare E. Hosseinzadeh S. [Effect of Groun Play Therany on Emotional/Behavioral \\
\hline 口prita & $\begin{array}{l}\text { Problems of Children With Attention Deficit Hyperactivity Disorder Aged 6-12 Years (Persian)]. Archives of Rehabilitation. 2020; } \\
\text { 21(3):390-405. https://doi.org/10.32598/RJ.21.3.3158.1 }\end{array}$ \\
\hline ablut & doi'https://doi.org/10.32598/RJ.21.3.3158.1 \\
\hline
\end{tabular}

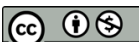

Received: 08 Jan 2020

Accepted: 14 Aug 2020

Available Online: 01 Oct 2020

Keywords:

Attention deficit and

hyperactivity disor-

der, Play therapy,

Behavioral problem

\section{ABSTRACT}

Objective One of the most significant issues in children with Attention Deficit Hyperactivity Disorder (ADHD) is emotional/behavioral problems, which disrupt their social communication at home and school. Some of these problems include social problems, rule-breaking behavior, and aggressive behavior. These problems, if left untreated in childhood, can cause problems in adulthood such as substance abuse, insubordination in the workplace, shorter stay at a job, and more anti-social acts. Nowadays, the use of play therapy for children with ADHD has become common. It is an active approach that allows the children to express their feelings through play. In group play therapy, children are asked to evaluate their personality according to their peers' reactions. The study investigates the effect of group play therapy on emotional/behavioral problems in children with ADHD aged 6-12 years.

Materials \& Methods This is a randomized controlled trial. The study population consists of all children with ADHD referred to the Dostdaran Koodak Occupational Therapy Clinic in Isfahan City, Iran. Of these, 28 children aged 6-12 years (average age: 8 years), including 8 girls and 20 boys, were selected using a convenience sampling technique. First, the study objective was explained to the parents, and children were then randomly assigned into the intervention and control groups. Considering error $(d)=3$, with $95 \%$ confidence level and $80 \%$ test power, the sample size was determined 14 for each group. The intervention group received 12 sessions of group play therapy ( 2 sessions per week, each for $45-60$ minutes), besides two sessions of individual occupational therapy per week. However, the control group received two sessions of occupational therapy per week only. The emotional/behavior problems of subjects in both groups were assessed before and after the intervention using the CBCL. Data analysis was performed in SPSS V. 21 software using the Shapiro-Wilk test and ANCOVA.

Results After the group play therapy, there was a significant decrease in the scores of emotional/behavioral problems, including social issues, aggressive behavior, and rule-breaking behavior, as well as in the overall score $(\mathrm{P}<0.001)$.

Conclusion Group play therapy can be used as a complementary method with other common occupational therapies for reducing the emotional/behavioral problems in children with ADHD.

\section{*Corresponding Author: \\ Hooshang Mirzai, PhD.}

Address: Department of Occupational Therapy, School of Rehabilitation Sciences, University of Social Welfare and Rehabilitation Sciences, Tehran, Iran. Tel: +98 (912) 2208426

E-Mail: hooshang_mirzaie@yahoo.com 


\section{Extended Abstract}

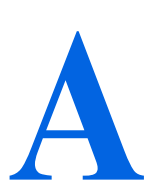

\section{Introduction}

ttention Deficit Hyperactivity Disorder (ADHD) is one of the most common disorders diagnosed in childhood and is associated with impulsivity and impaired attention $[1,2]$. According to the fifth edition of the Diagnostic and Statistical Manual of Mental Disorders (DSM-5), the prevalence of $\mathrm{ADHD}$ is $5 \%$ in children and $2.5 \%$ in adults [1]. Its prevalence in Iranian children is 3\%-7\% [3]. The DSM-5 has listed 18 symptoms for ADHD, and at least 6 symptoms are required for its diagnosis. Depending on these symptoms, ADHD has three types: predominantly impulsive/hyperactive, predominantly inattentive, and the combined type [1]. Emotional/behavioral disorders in children with ADHD are manifested in two general forms of externalizing symptoms (e.g. stubbornness, aggression, lawlessness) or internalizing symptoms (e.g. isolation, depression, and anxiety).

Group play therapy helps to improve children's leadership and social skills [5]. It is effective in reducing children's emotional/behavioral problems [6-15]. To our knowledge, few studies in Iran have used group play therapy. Also, since group therapy is one of the subjects of occupational therapists, they have intervened less in this field. This study investigates the effect of group play therapy on emotional/ behavioral problems in children with ADHD.

\section{Materials and Methods}

This research is a randomized controlled clinical trial. The study population consists of all children with ADHD referred to Dostdaran Koodak Occupational Therapy Clinic in Isfahan City, Iran. Those with an average age of 8 years, including 8 girls and 20 boys, were selected as study samples using a convenience sampling technique. They were then randomly assigned to the intervention and control groups. The sample size [16] for each group was determined 14 . The inclusion criteria were diagnosis of ADHD by a child psychiatrist, completion of written informed consent by the parents, aged between 6 and 12 years, a Child Behavior Check List (CBCL) score $>60$, no psychotic symptoms according to the Child Symptom Inventory-4 (CSI-4), and no previous history of participation in similar studies. The exclusion criteria were the absence of more than 2 sessions from the intervention and lack of parental cooperation.

The data collection tools were CSI- 4 and CBCL. The CSI4 items are never $=0$, sometimes $=0$, often $=1$, and always $=1$ [17]. Esmaeil, in a study on 715 normal students and 419 students with ADHD in Tehran, Iran, evaluated the psycho- metric properties of the Persian CSI-4 [18]. He reported internal consistency of 0.76 for the predominant types of $\mathrm{ADHD}$ and 0.81 for the combined type of ADHD, and high criterion validity of 0.8 for most subscales. The CBCL measures 8 symptoms of anxious/depressed, withdrawndepressed, somatic complaints, social problems, thought problems, attention problems, rule-breaking behavior, and aggressive behavior [16]. The CBCL consists of 113 questions, measuring the children's symptoms within the past 6 months scored on a 3-point Likert scale (not true $=0$, sometimes true $=1$, often true $=2$ ) [19].

After obtaining ethical approval and an introductory letter from the University of Social Welfare and Rehabilitation Sciences, and permission from the clinic, the samples were selected. Their parents signed the informed consent form and completed the demographic form, CSI-4, and CBCL. Then, they were randomly divided into two groups. Besides receiving 2 sessions of individual occupational therapy per week, the intervention group participated in group play therapy in 12 sessions ( 6 weeks, 2 sessions per week, each for 45-60 minutes). The control group received only 2 sessions of individual occupational therapy per week. After 12 sessions [20], the CBCL was filled out by the children's parents again.

\section{Results}

The Mean \pm SD ages of children in the intervention and control groups were $8.1 \pm 1.6$ and $8.0 \pm 1.8$ years, respectively, and there was no significant difference between the two groups in terms of age. There were 5 girls $(35.7 \%)$ and 9 boys $(64.3 \%)$ in the intervention group and 3 girls $(21.4 \%)$ and $11(78.6 \%)$ boys in the control group.

As shown in Table 1, the mean scores of the two groups in the variables of social problems, lawlessness, and aggression in the pre-test stage were not significantly different, but in the post-test stage, the scores showed a significant difference. To test the effectiveness of group play therapy on emotional/behavioral problems in children, ANCOVA was used. First, the assumption of normality was checked using the Shapiro-Wilk test. Based on the results, there was normal data distribution $(\mathrm{P}>0.05)$. As seen in Table 2 , in the social issues variable, group factors and pre-test scores significantly affected post-test scores $(\mathrm{P}<0.05)$. The effect of the intervention and pre-test score for the social issues variable were 0.82 and 0.84 , respectively. This result means that the group play therapy and the pre-test social problems score could explain $82 \%$ and $84 \%$ of changes in the post-test social problems score, respectively. For rulebreaking behavior, group factors and pre-test scores also significantly affected post-test scores $(\mathrm{P}<0.05)$. The effect 
Table 1. Descriptive statistics of demographic characteristics and emotional/behavioral problems of children

\begin{tabular}{cccc}
\hline & & \multicolumn{2}{c}{ Mean \pm SD } \\
Variables & Group & Pre-test & Post-test \\
\cline { 3 - 4 } Social problems & Intervention & $9.5 \pm 3.1$ & $3.0 \pm 2.7$ \\
& Control & $10.1 \pm 2.8$ & $7.7 \pm 4.7$ \\
\hline Rule-breaking behavior & Intervention & $8.9 \pm 2.5$ & $4.1 \pm 1.5$ \\
& Control & $7.0 \pm 2.8$ & $6.5 \pm 2.8$ \\
\hline Aggressive behavior & Intervention & $25.0 \pm 4.8$ & $9.9 \pm 3.2$ \\
& Control & $20.0 \pm 6.8$ & $19.8 \pm 5.9$
\end{tabular}

Table 2. Test of between-subjects effects using ANCOVA

\begin{tabular}{cccccccc}
\hline Dependent Variable (post-test) & Independent Variable & Sum of Squares & df & $\begin{array}{c}\text { Mean } \\
\text { Square }\end{array}$ & F & $\begin{array}{c}\text { P } \\
\text { Squared }\end{array}$ & $\begin{array}{c}\text { Partial Eta } \\
\text { Square }\end{array}$ \\
\hline Social problems & Pre-test score & 91.4 & 1 & 91.4 & 95.5 & $<0.001$ & 0.841 \\
& Group & 78.5 & 1 & 78.5 & 82 & $<0.001$ & 0.82 \\
Rule-breaking behavior & Pre-test score & 39.5 & 1 & 39.5 & 31.2 & $<0.001$ & 0.635 \\
& Group & 78.5 & 1 & 78.5 & 62.1 & $<0.001$ & 0.775 \\
Aggressive behavior & Pre-test score & 91 & 1 & 91 & 40.9 & $<0.001$ & 0.695 \\
& Group & 825.7 & 1 & 825.7 & 371.2 & $<0.001$ & 0.954 \\
\hline
\end{tabular}

Rehabilitation

of the intervention and pre-test score for rule-breaking behavior were 0.78 and 0.63 , respectively. This result means that the group play therapy and the pre-test rule-breaking behavior score could explain $78 \%$ and $63 \%$ of changes in the post-test rule-breaking behavior score, respectively. For aggressive behavior, group factors and pre-test scores also significantly affected post-test scores $(\mathrm{P}<0.05)$. The effect of the intervention and pre-test score for aggressive behavior were 0.95 and 0.7 , respectively. This result means that the group play therapy and the pre-test aggressive behavior score could explain $95 \%$ and $70 \%$ of changes in the posttest aggressive behavior score, respectively. Overall, it can be said that group play therapy significantly affects social problems, rule-breaking behavior, and aggressive behavior of children with ADHD (Figures 1, 2, and 3).

\section{Discussion and conclusion}

One study aimed to investigate the effect of group play therapy on the social problems of children with ADHD [21]. The scores of social issues over time showed a significant downtrend in both intervention and control groups. Comparing the two groups showed a significant difference between them where the decreasing trend was more in the intervention group than in the control group. This result is consistent with the results of Naderi et al. Soleimany, Karami, Cheng, Hensen et al. and Rosenberg et al. [15, 22-26]. According to Naderi et al. group play therapy is a suitable intervention for children who experience various problems. Group play therapy is an intervention that is not dangerous for children, and many studies have confirmed this approach. In this study, play therapy was an effective intervention for the social maturity of children with ADHD. 


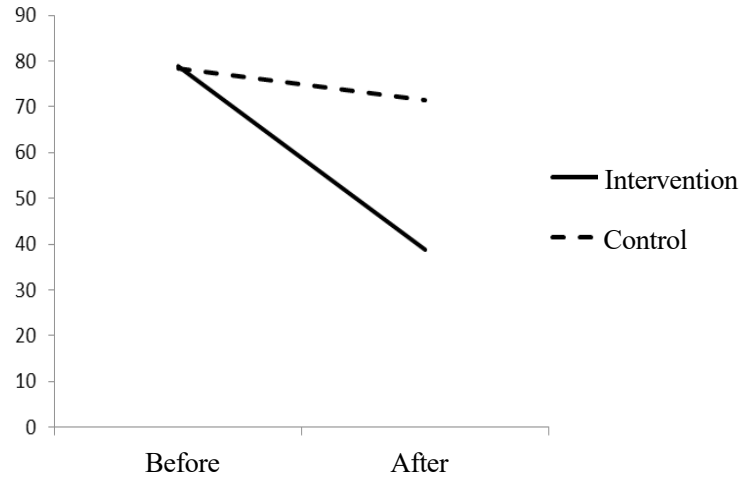

Rehabilitation

Figure 1. Comparing the mean score of social problems in two study groups before and after the intervention

However, it was not used in the study by Naderi et al. [42]. In Soleimany's research, which was quasi-experimental, he used rhythmic games, which finally led to improved social skills in children with ADHD [43]. Cheng, in a clinical trial, evaluated the impact of child-centered group play therapy on social-emotional assets of kindergarten children. After receiving the intervention, children made significant progress in empathy and social adequacy [16]. In Hensen et al. study, group play therapy also improved the social skills of children with ADHD [25].

Two other purposes of this study were to investigate the effect of group play therapy on rule-breaking behavior and aggressive behavior of children with ADHD. The results showed a significant decrease in the score of these behaviors in both groups after the intervention, where the decreasing trend was more in the intervention group than in the control group. This finding is consistent with the results of Almeraisi [46]. He used cognitive-behavioral play therapy on children with ADHD, which reduced their aggressive behaviors. Our result regarding the reduction of aggressive behavior is also consistent with Ray et al. [19]. Smith [22] used group play therapy for children with ADHD and witnesses of domestic violence to reduce their rule-breaking and aggression behaviors, respectively and reported its positive effects. However, in these studies, the impact of group and individual play therapy was assessed equally, and there was no significant difference between them. Azadimanesh used puppet play therapy, and Kahrizi applied sand play therapy to reduce the aggression of children with ADHD [48, 49].

From an observational and clinical perspective, children's emotional and behavioral problems were very high before group play therapy in our study. The children were so anxious that it affected their social participation. The aggression level also increased, and the children were very aggressive towards each other in the first sessions, and there

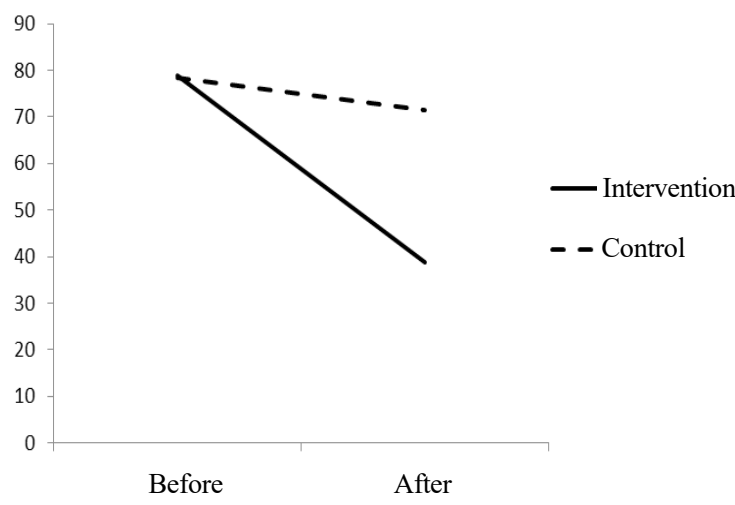

Rehabilitation

Figure 2. Comparing the mean score of rule-breaking behavior in two study groups before and after the intervention

was an incompatibility between them. Some children disregarded the rules of the group therapy and tried to play their games. After the intervention, significant changes were observed in them such that their anxiety level decreased and social communication improved. Their violence and aggression towards each other also reduced, and they followed the rules of the group better. Parents also reported an overall improvement in their children's relationship with classmates and teachers after the intervention. According to them, the children's participation in the classroom, social interaction, obedience at home, and participation in individual occupational therapy sessions also increased.

Group play therapy can be used as a complementary method and other common occupational therapy methods for reducing the emotional/behavioral problems of children aged 6-12 years with ADHD. The main limitations of the present study were sampled by a convenience sampling method, time limitation of group play therapy, and lack of follow-up assessment. In future studies, it is recommended

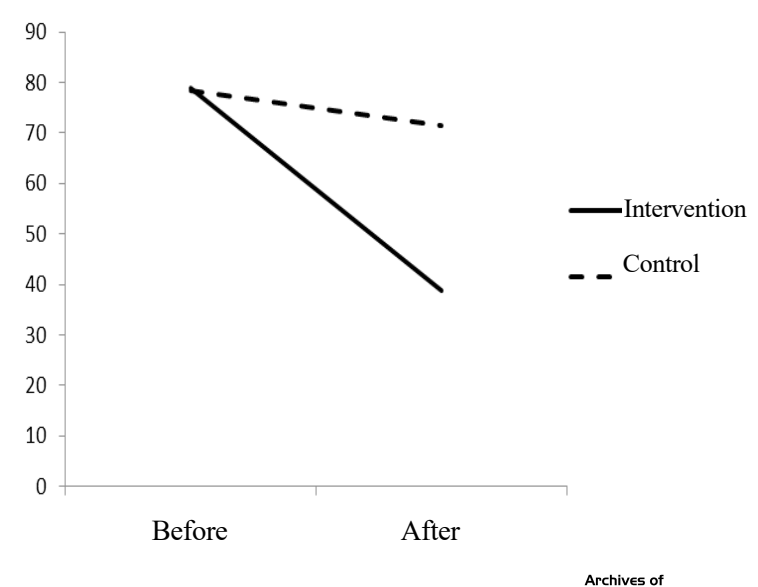

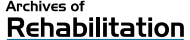

Figure 3. Comparing the mean score of aggressive behavior in two study groups before and after the intervention 
to pay attention to the durability of treatment effect after the intervention and the long-term impact of the intervention on behavioral disorders of these children.

\section{Ethical Considerations}

Compliance with ethical guidelines

This study is a registered clinical trial (Code: IRCT20190924044875N1) and has ethical approval from the Research Ethics Committee of the University of Social Welfare and Rehabilitation Sciences (Code: IR.USWR. REC.1397.089).

Funding

The present paper was extracted from the MSc. thesis of the first author, Department of Occupational Therapy, School of Rehabilitation, University of Social Welfare and Rehabilitation Sciences.

Authors' contributions

Ideation and conceptualization: Houshang Mirzaei and Sepide Teimouriani; Research analysis: All authors; Review of sources: Ebrahim Pishyare, Sepide Teimourian; Drafting, editing and finalization: Houshang Mirzaei, Ebrahim Pishyare, Sepide Teimourian; Supervision: Houshang Mirzaei, Ebrahim Pishyare, Samaneh Hosseinzadeh; Management: Houshang Mirzaei.

Conflict of interest

The authors declared no conflict of interest

Acknowledgments

The authors would like to thank the staffs of Tohid occupational therapy clinics. 


\title{
تأثير بازى ادرمانى گروهى بر مشكلات اجتماعى، يرخاشحَرى وقانونشكنى كودكان شش تادوازده سال با نقص توجه و بيش فيشعالى بروفي
}

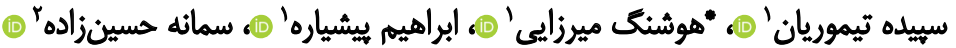 \\ ا ا.كروه كاردرمانى، دانشكده علوم توانبخشى، دانشكاه علوم بهزيستى و توانبخشى، تهران، ايران. \\ ب. كروه آمار زيستى، دانشكده علوم توانبخشى، دانشكاه علوم بهزيستى و توانبخشى، تهران، ايراني.
}

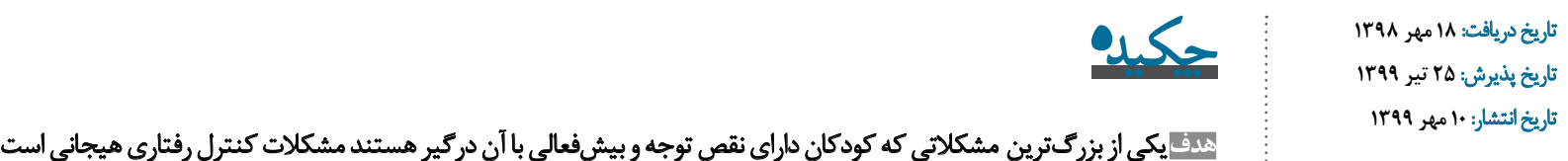

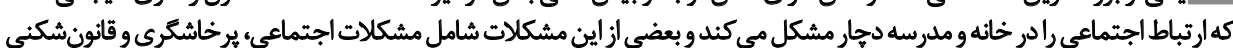

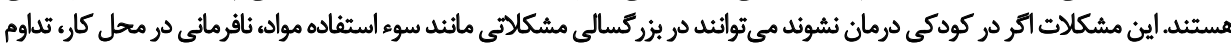

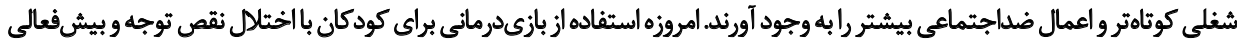

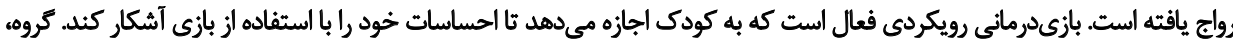

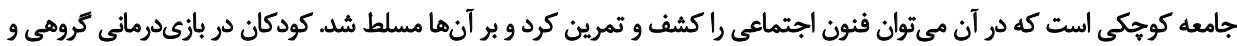

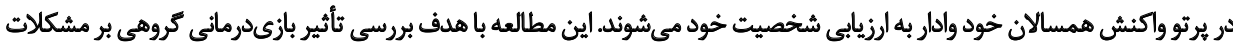

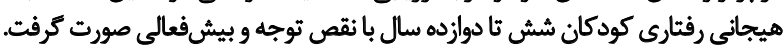

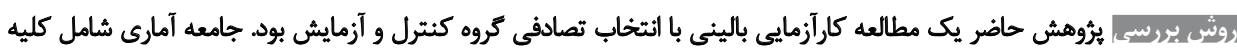

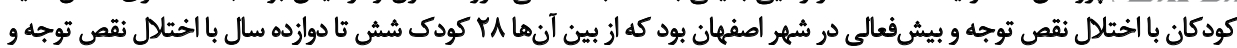

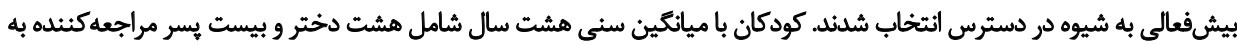

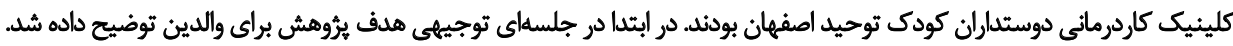

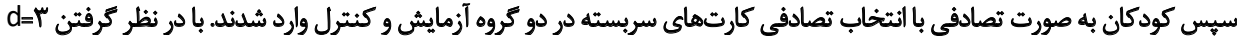

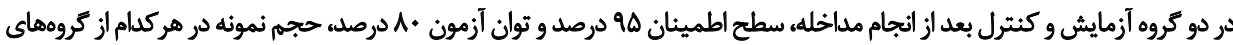

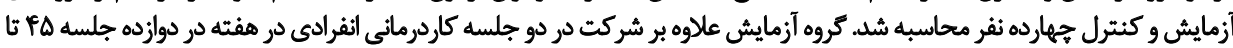

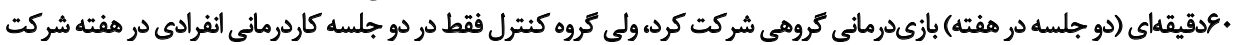

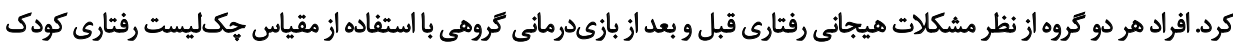

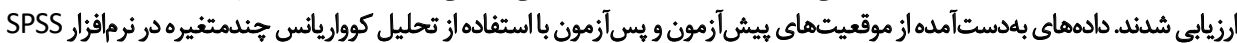
نسخه آ تجزيه و تحليل شديند

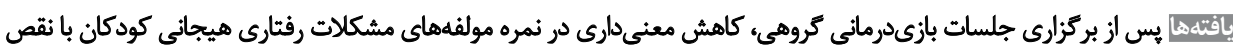

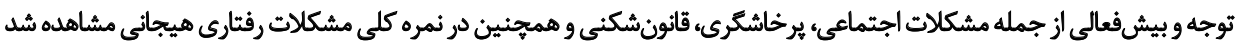
(P< 1.01$)$

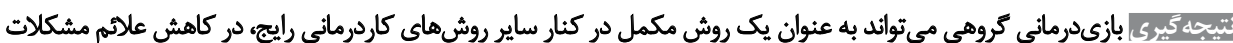

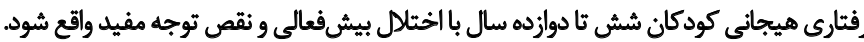

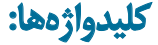

اختلال نقص توجه و

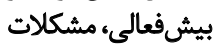
هيجانى رفتارى، باريكات

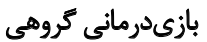


[ [1]. درمان نقص توجه و بيشفعالى در سنين يهيش از دبستان

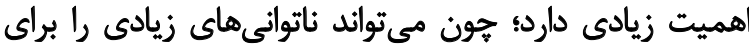
كودى و خانوادهاش ايجاد كند [11]

كودكان داراي اختلال نقص توجه و بيشفعالى با مشكل آنساري

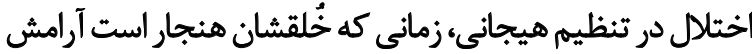

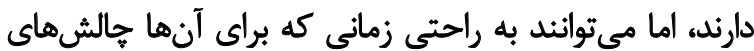

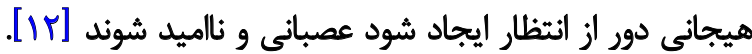

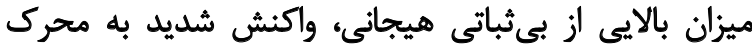

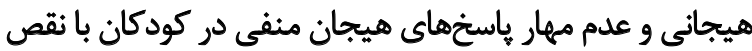

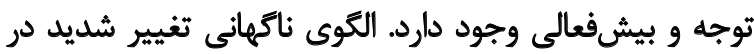

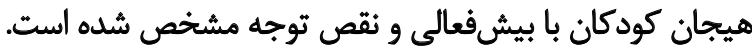

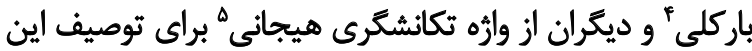

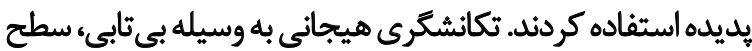

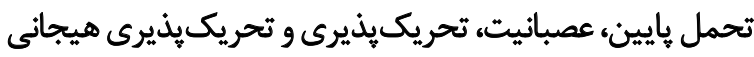
مشخص مىشود [آسايت.

بازىدرمانى گروهى به بهبود مهارتهاى رهبرى در كودكان

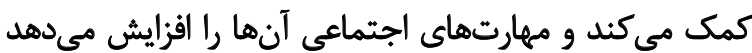

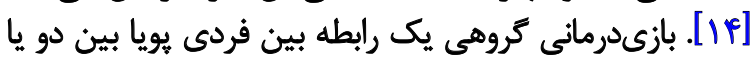

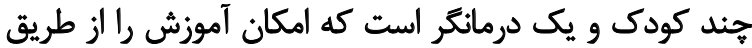

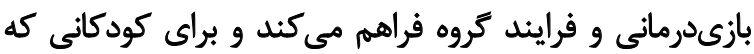

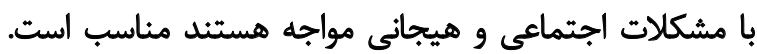

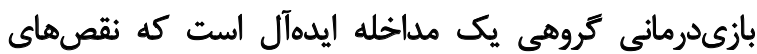

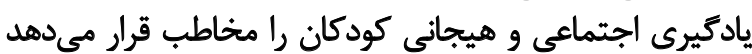

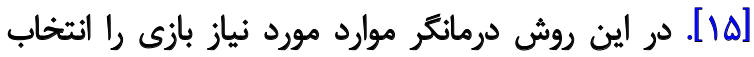

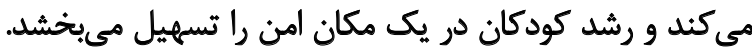

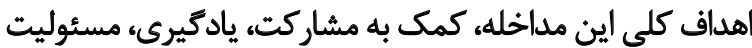

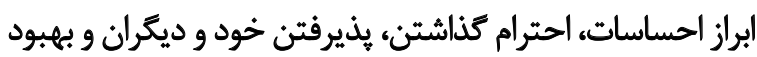

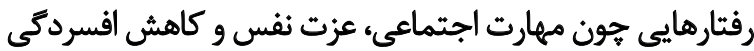

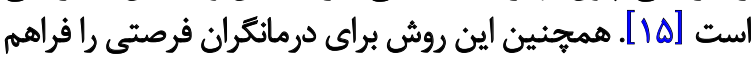

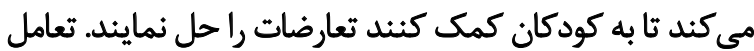

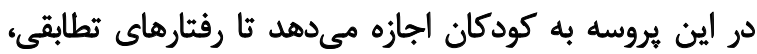

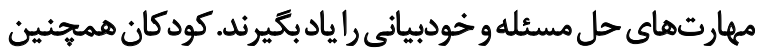

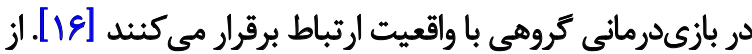

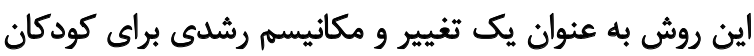

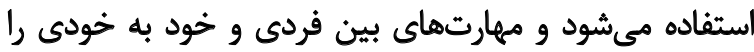

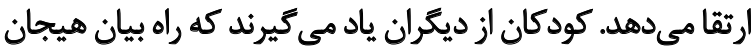

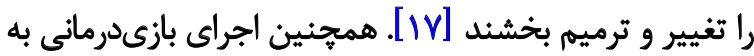

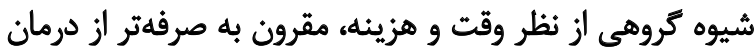

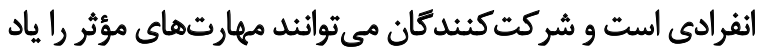

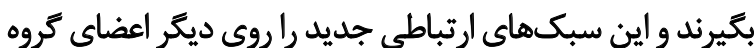
امتحان كنئد [1/] مelat

بيش فعالى و نقص توجه يكى از اختلالات شايع دوران كودكى دي

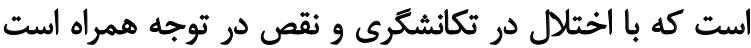

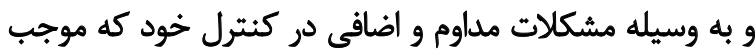

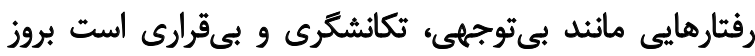

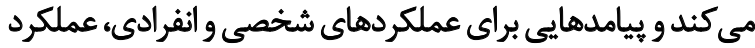

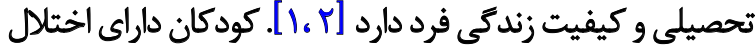

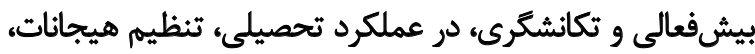

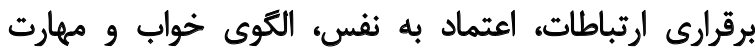

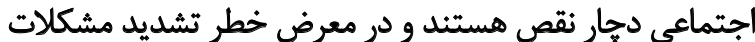

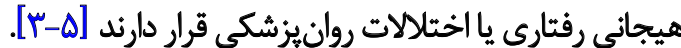

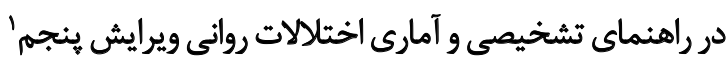

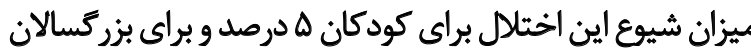

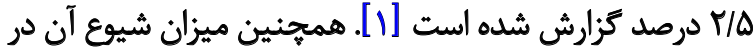

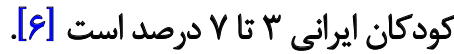

هيجده نشانه براى اين بيمارى در DSM-5 ارائه شده كه

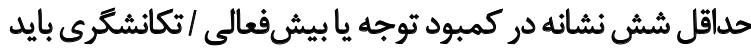

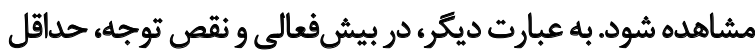

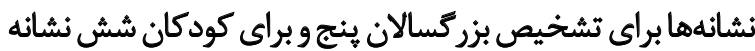

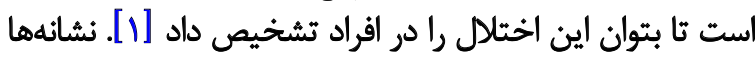

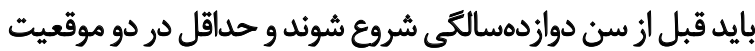

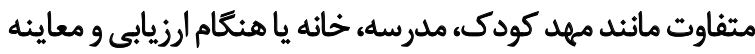

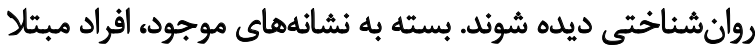

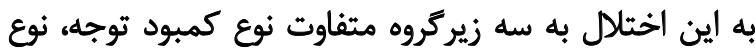

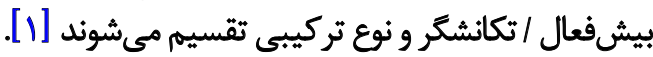
كودكان بيشفعال نسبت به شرايط هيجاني واكنش بيش از حد

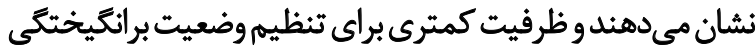

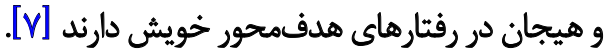

اختلالات هيجانى رفتارى در كودكان با بيشفالى ونالى و نقص

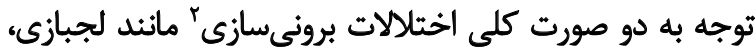

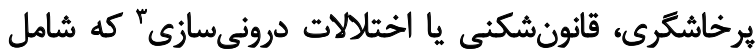

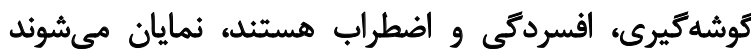

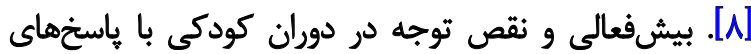

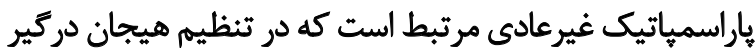

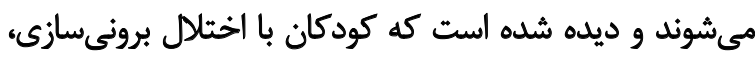

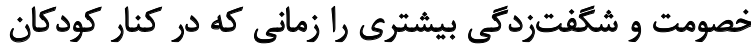

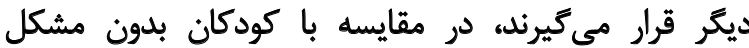

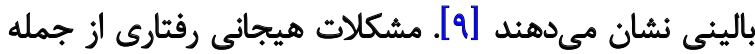

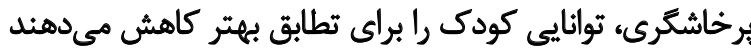

1. Diagnostic and Statistical Manual of Mental Disorder-5 (DSM-5) 2. Externalization

3. Internalization 


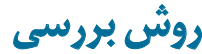

يُروهش حاضر، يك مطالعه كارآزمايى بالينى كنترلشده

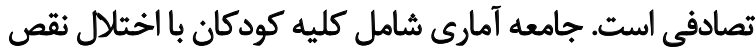

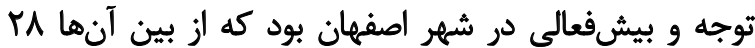

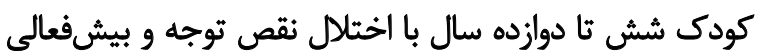

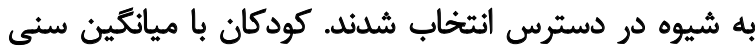

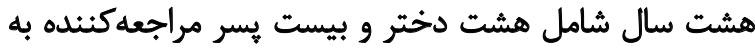

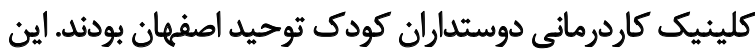

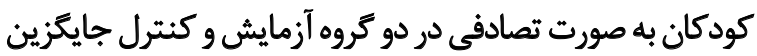

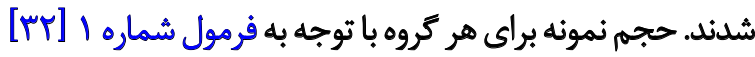
جهارده نفر تعيين شد:

\section{.1}

\section{$n=\left((z(1-\alpha / 2)+z(1-6))^{2}\left(s 1^{2}+s 2^{2}\right)\right) / d^{2} \cong 14$}

ملاكهاى ورود به مطالعه شامل تشخيص اختلال نقص توجه

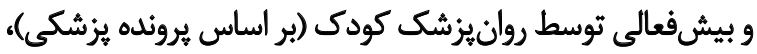

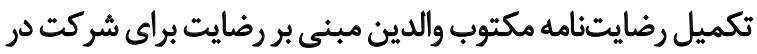

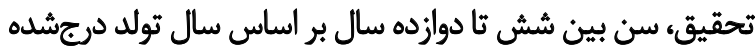

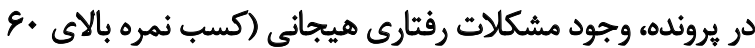

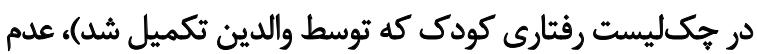
وجود علائم بارز سايكوتيك در كودى (آزمون

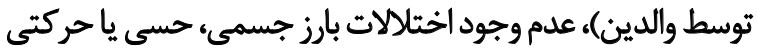

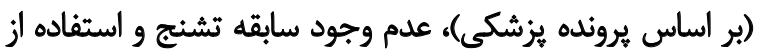

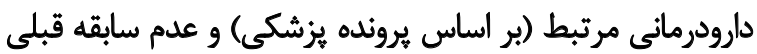

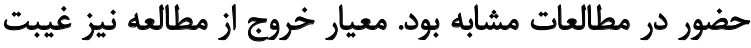
بيش از دو جلسه و عدم همكارى والدين در نظر كرفته شد. ابرزار يُروهش شامل يرسشنامه عائائم مرضى كودكان و جكليست رفتارى كودك بود:

\section{"ير سش بامه عالائم مرضى كودكان"}

از اين برسشنامه كه توسط اسيرافكين و وكادو^ ساخته شده

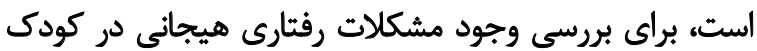

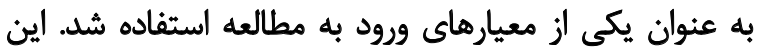

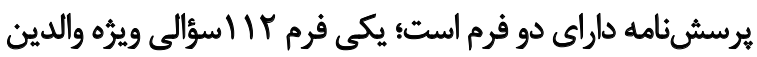

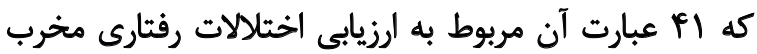

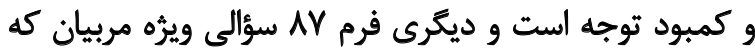

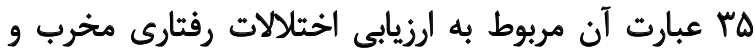

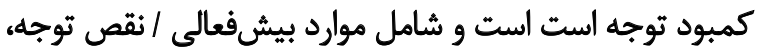

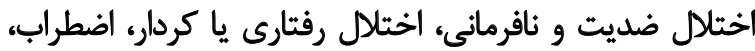

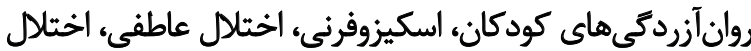

6. Child Symptom Inventory-4 (CSI-4)

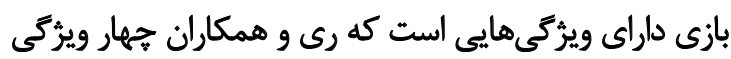

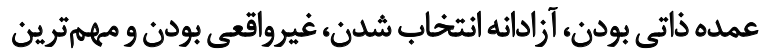

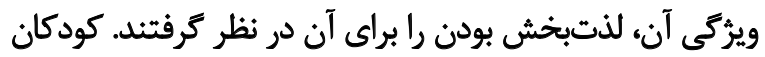

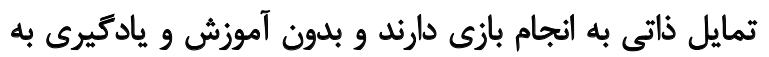

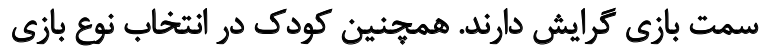

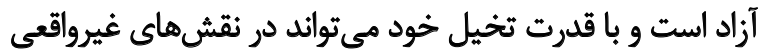
بازى حاضر شود [19].

بازى، دستهبندى هاى مختلفى دارد كه يكى ازئ اين دستهبندىها

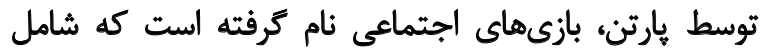

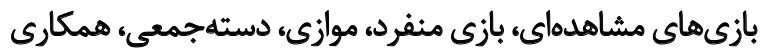

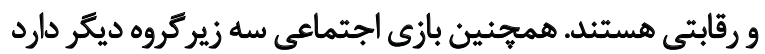

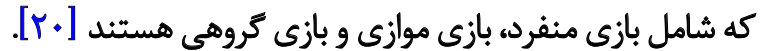

طبقهبندى ديكر انواع بازى، دستهبندى اندرسون مكنيم است

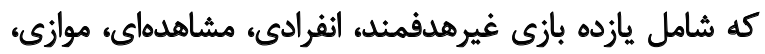

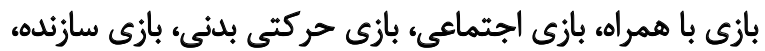

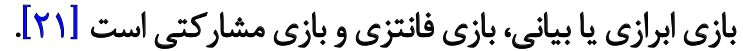

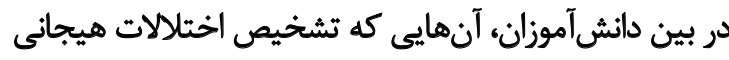

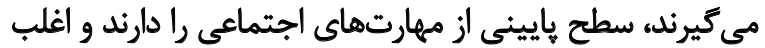

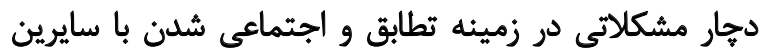

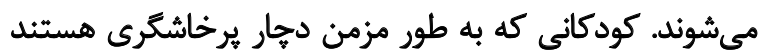

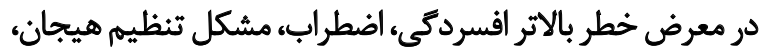

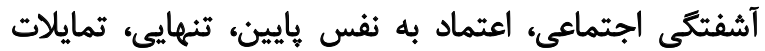

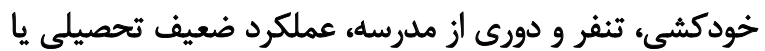

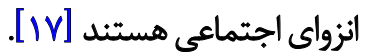

مطالعات نشان مىدهند بازىدرمانى كروهى در مرومهان

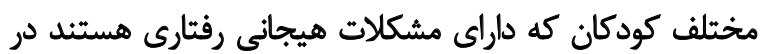

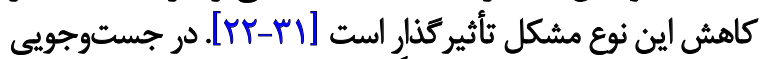

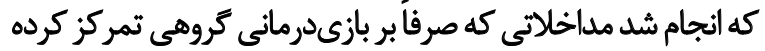

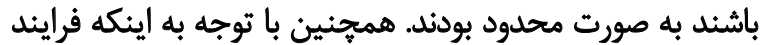

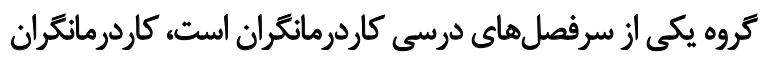

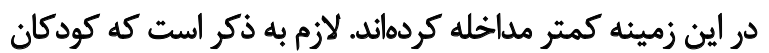

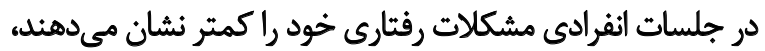

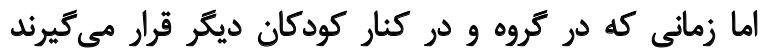

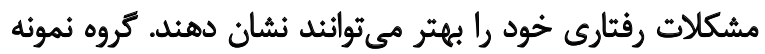

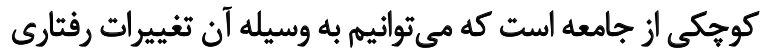

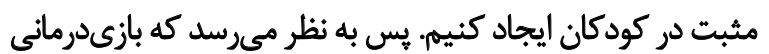

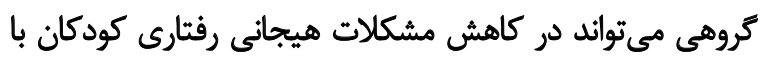

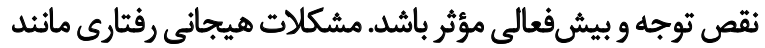

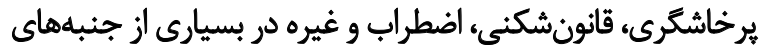

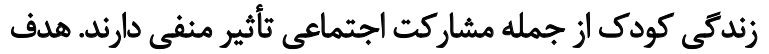

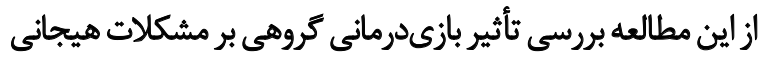

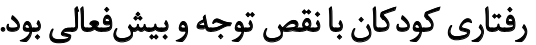




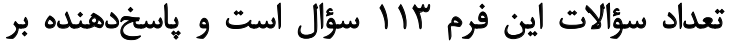
اساس وضعيث كودى در شش ماه كذشته هر سؤال را باله صورت

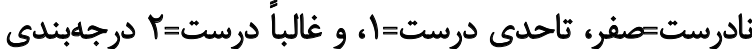

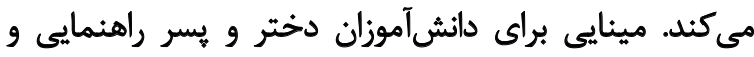

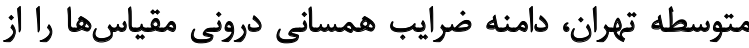

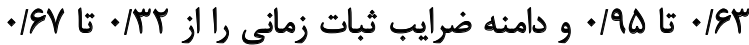

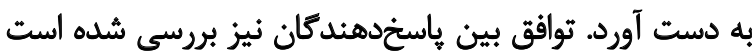

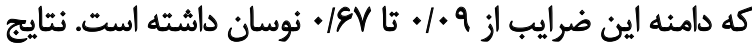
بهدستآمده از بررسى روايى محتوايیى، سازهاي و ملاكى نيز

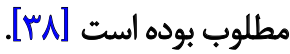

يزدخواستى و عريضى براي دانش آموزان دبستانى دختر و پيسر

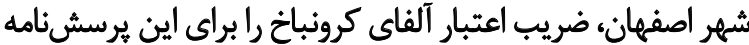

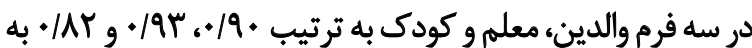

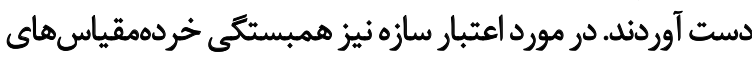

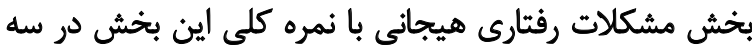

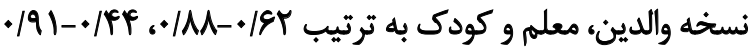

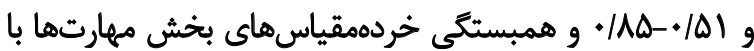

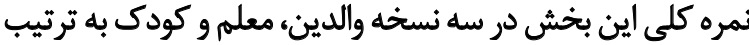

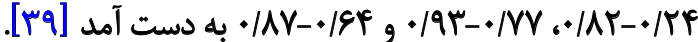

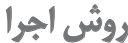

اين يُؤوهش با شناسه

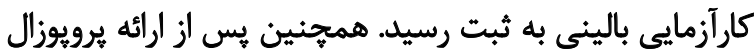

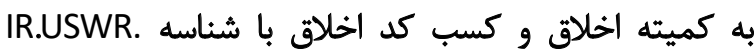

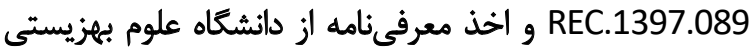
و توانبخشى جهت هماهنكى با مسئولين كلينيك، اجازه اندام اندام

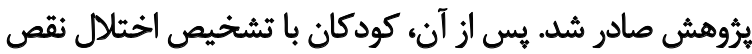

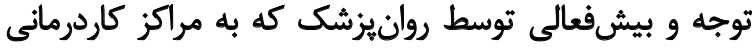

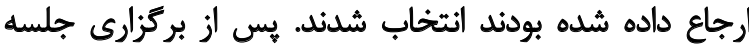

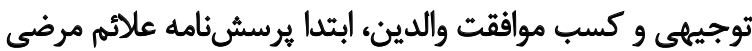

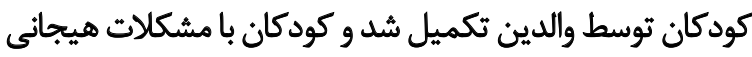

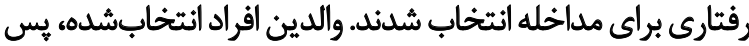

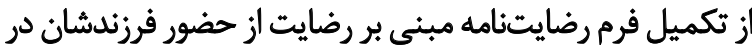

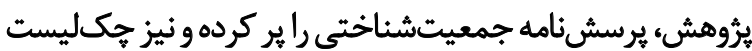

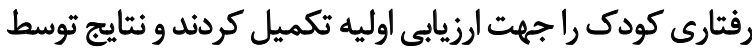

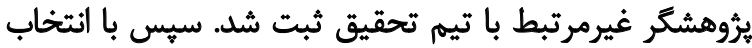

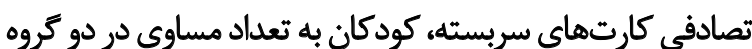

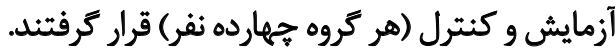

كروه آزمايش علاوه بر دريافت دو جلسه كاردرمانى انفرادى

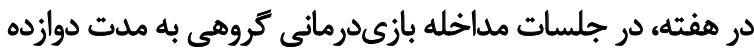

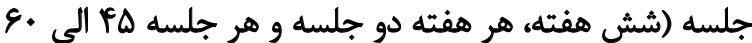

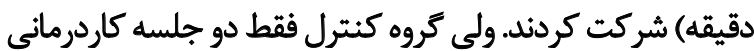

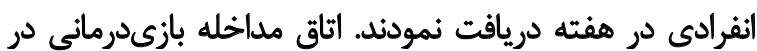

درخودماندگى و ترسهاى مرضى اجتماعى هي فيود كه در اين

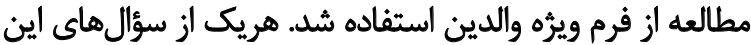

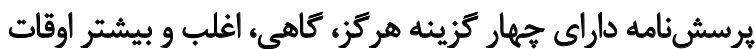

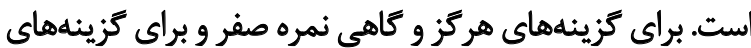

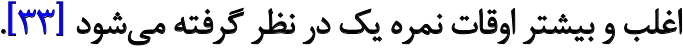

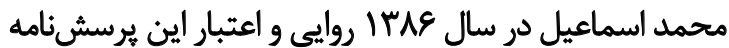

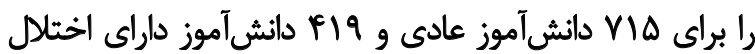

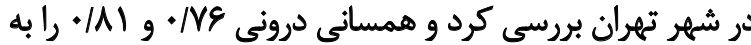

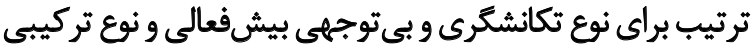

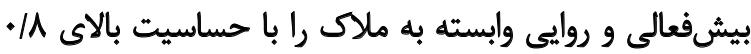

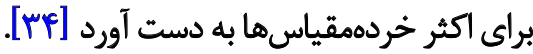

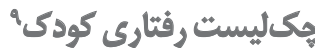

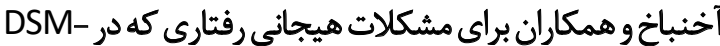

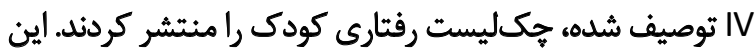

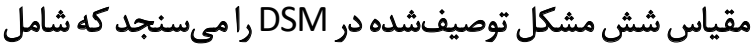

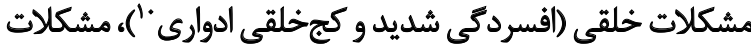

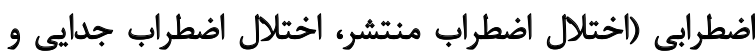

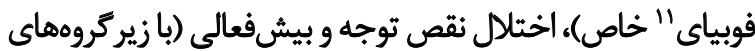
بيشفعال تكانشكر و بي توجهى)، اختالات اتلات رفتارى، مشكلات لجاجت و مشكلات جسمى هستند [ه"آ]

جكىليست رفتارى كودك، آزمونى استاندارد است كه والدين

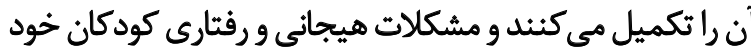

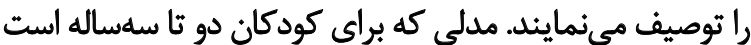

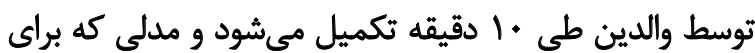

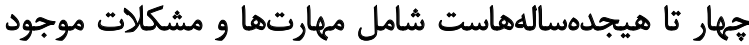

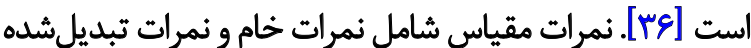

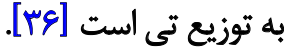

اين مقياس شامل دو بعد وسيع مشكلات بيرونى و درونى و و

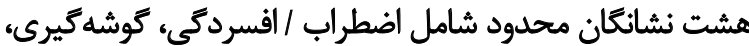

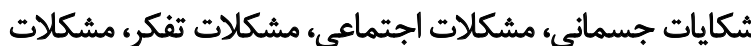

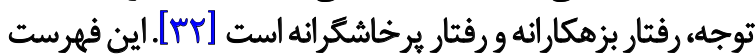

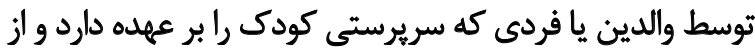

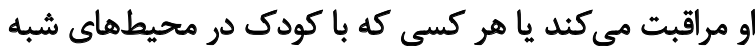

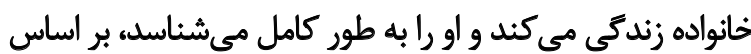

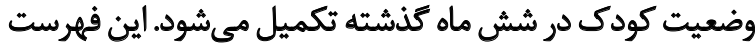

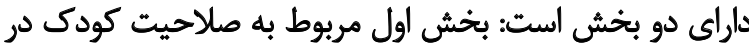

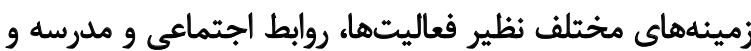

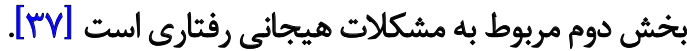

9. Child Behavioral Check List (CBCL)

10. Dystimya

11. Phobia 
مرحله سوم: همكارى (جلسات ششم ثأ يازدهم) شامل انجام

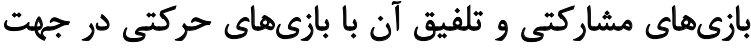

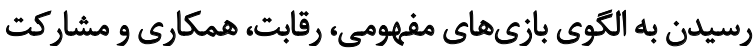

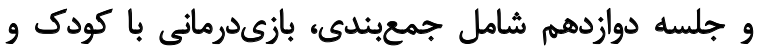

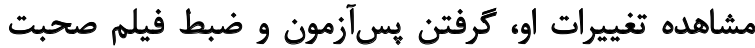

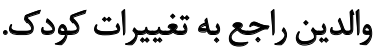

Latọ

آزمودنىها شامل Y كودى (دختر و يسر) داراى اختلال

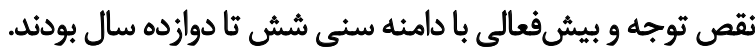
ميانكين سنى در كروه آزمايش بـ

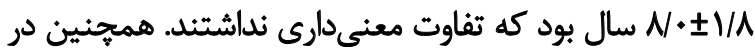

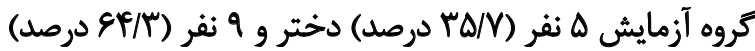

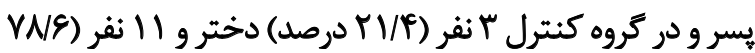

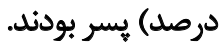

ميانكين نمرات دو كروه در متغير هاى اجتماعي، قانونشكنى و ئرو

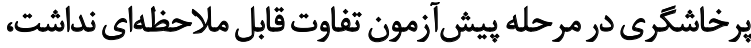

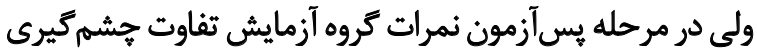

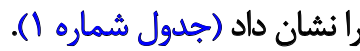

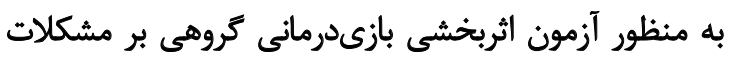

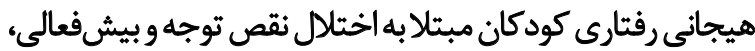

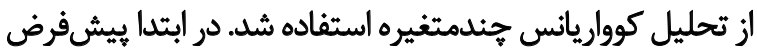

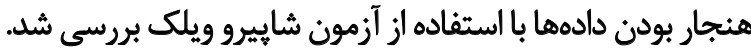

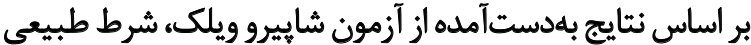

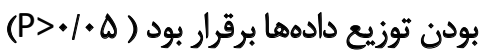
همانطور كه در جدول شماره Y ديده مي كيود در متغير

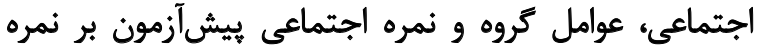

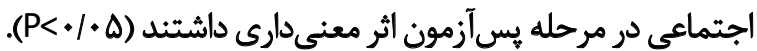

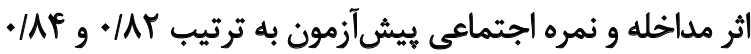

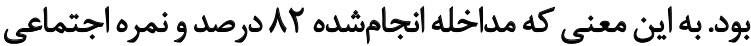

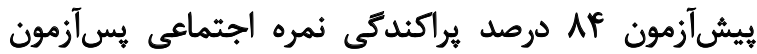

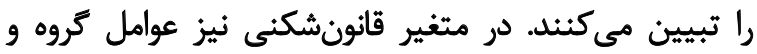

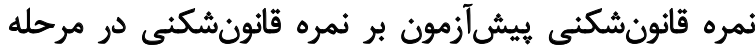

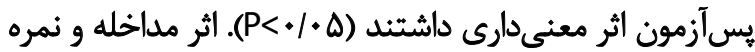

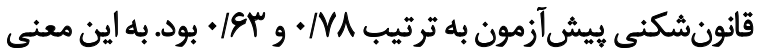

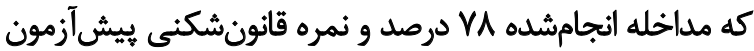

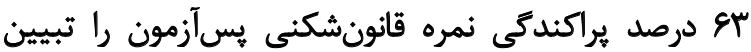

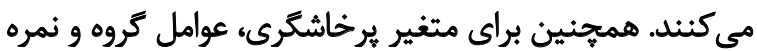

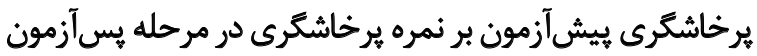

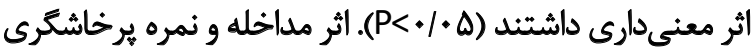

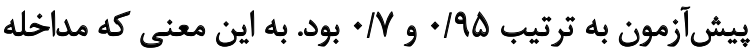

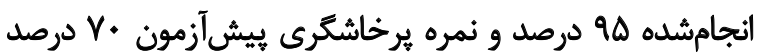

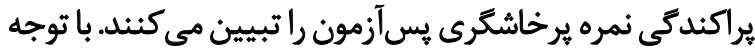

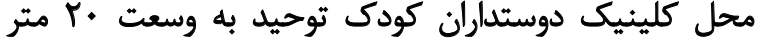

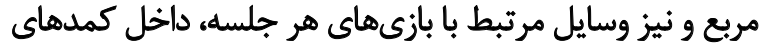

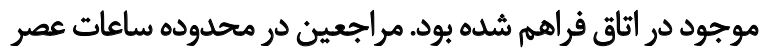

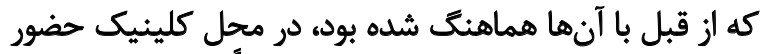

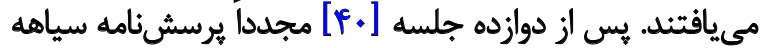
رفتارى كودكان توسط والدين تكميل شد.

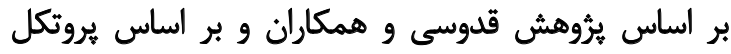

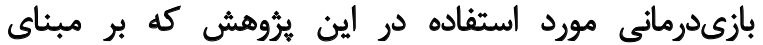

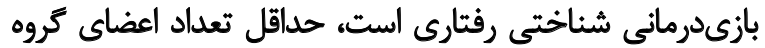

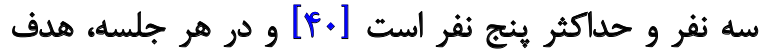

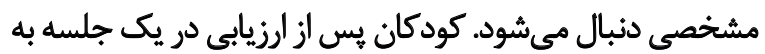

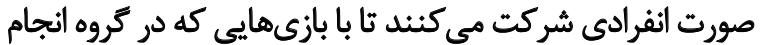
مي مود آشنايى لازم راكسب كنيند.

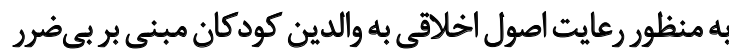

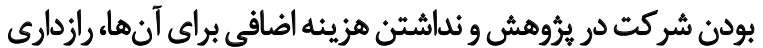

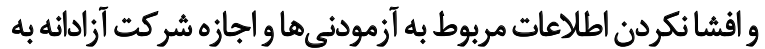

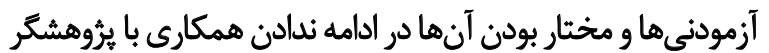

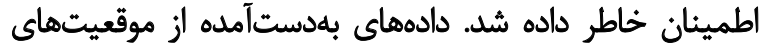

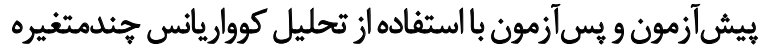
در نرمافزار SPSS نسخه آب تجزيه و تحليل شدادي

ساختّار جلسات درماني

فرايند بازىدرمانى در يازده كام يَيكيرى شد كه عبارت بودند

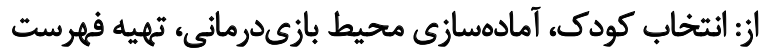

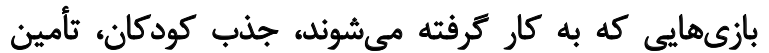

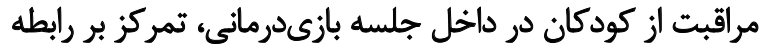

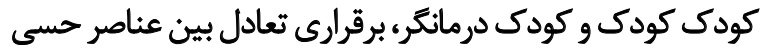

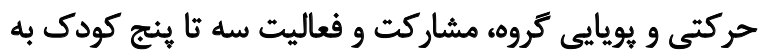

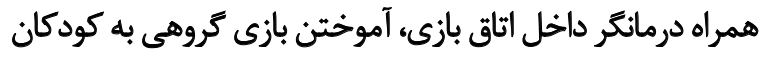

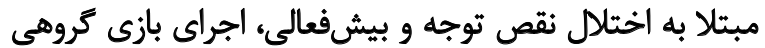

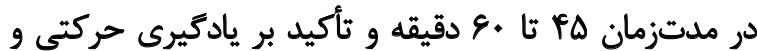
قاعدهمند بودن بازى.

ساختار جلسات درمانى در يروهش حاضر به شرح زير بود:

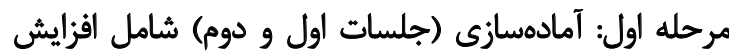

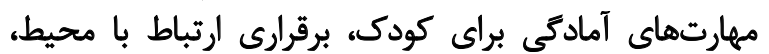

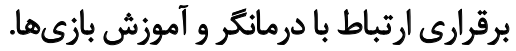
مرحله دوم: همتاسازى (جلسات سوم تا ينجمم) شامل آغاز

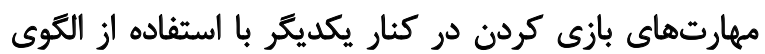

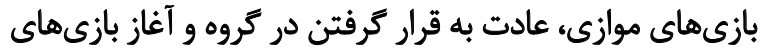

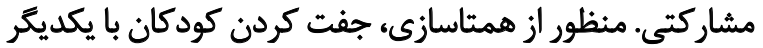
به منظور انجام بازى دونفره است. 
جدول ا. شاخصهاى توصيفى متغيرهاى اجتماعى، قانونشكنى و يرخاشكرى در يبشآزمون و يس آزمون در دو كروه آزمايش و كنترل

\begin{tabular}{|c|c|c|c|}
\hline \multicolumn{2}{|c|}{ ميانكين Iانحرافمعيار } & \multirow{2}{*}{ ت تروه } & \multirow{2}{*}{ متغيرها } \\
\hline بس أزمون & ييش أزمون & & \\
\hline$r / \pm Y / N$ & $\checkmark / \Delta \pm r / /$ & آزمايش & \multirow{2}{*}{ الجتماعى } \\
\hline$Y / N \pm * / V$ & $1 . / \pm \pm r / A$ & كتترل & \\
\hline$\varphi / / \pm V / \Delta$ & $N q \pm r / \Delta$ & أزمايش & \multirow{2}{*}{ تأونشكنى } \\
\hline$s / \Delta \pm r / A$ & $V /= \pm r / A$ & كنترل & \\
\hline $9 / 9 \pm r / r$ & $\Gamma \Delta / \pm \pm r / A$ & آزمايش & \multirow{2}{*}{ ل برخاشكرى } \\
\hline $19 / 1 \pm \Delta / q$ & $r+/ \pm g / A$ & كنترل & \\
\hline
\end{tabular}

جدول r. نتايج تحليل كوواريانس جندمتغيره براى خردمقياسهاى اجتماعى، قانونشكنى و برخاشكرى آزمون سياهه رفتارى كودكان داراى اختلال نقص توجه و بيشفعالى

\begin{tabular}{|c|c|c|c|c|c|c|c|}
\hline اندازه اثر مجذور اتا & معنى سطرى & F أماره آزمون F & ميانكين مربعات & درجه آزادى & مجموع مربعات & متغير مستقل & متغير وابسته (يسأزمون) \\
\hline - $|A F|$ & $<+/ .+1$ & $9 \Delta / \Delta$ & $91 / 4$ & 1 & $91 / \%$ & اجتماعى بيشآزمون & \multirow{2}{*}{ 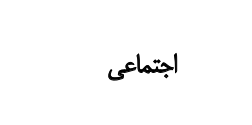 } \\
\hline /AT & $<+/ .+1$ & Ar & WND & 1 & YND & كروه & \\
\hline 每 & $<+1 *+1$ & $r / r$ & $r q / \Delta$ & 1 & $r q / \Delta$ & قانونشكنى بيش أزمون & \multirow{2}{*}{ ق قانونشكنى } \\
\hline.$/ W \Delta$ & $<.1 . .1$ & $8 / 1$ & VND & 1 & VND & كووه & \\
\hline .1998 & $<+1 .+1$ & $p \cdot / q$ & १७/. & 1 & 91\% & برخاشكرى ييش أزمون & \multirow{2}{*}{ يرخاشكرى } \\
\hline.$/ 9 \Delta f$ & $<+/+\infty 1$ & $r w / r$ & $\Delta T \Delta / Y$ & 1 & $\Delta T \Delta / Y$ & كروه & \\
\hline
\end{tabular}

مداخلهاى مناسب براى كودكانى است كهة مشكلات متنوعى راتجربه

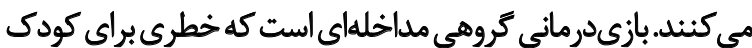

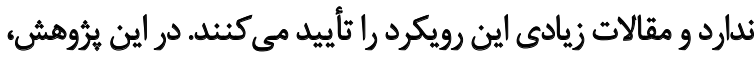

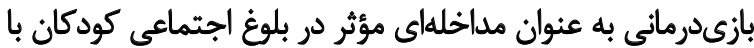

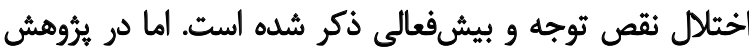

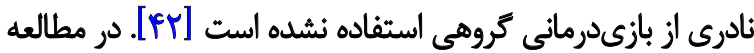

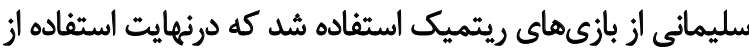

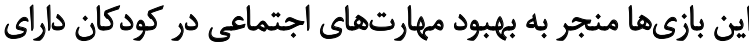

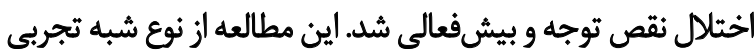

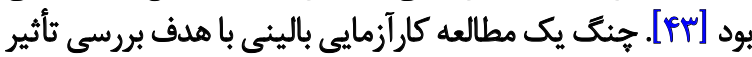

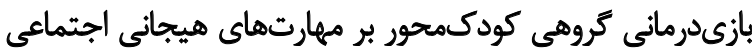

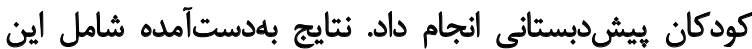

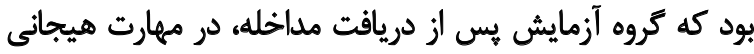

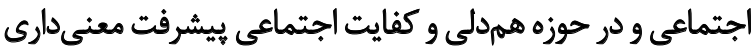

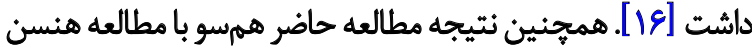

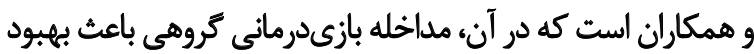

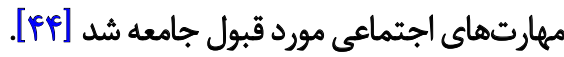

به اينكه نمرات افراد نمونه در متغيرهاى مشكلات اجتماعى،

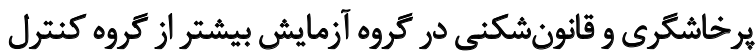

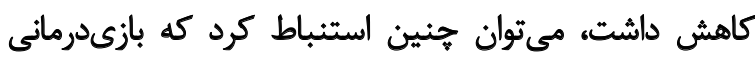

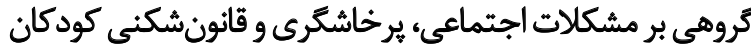

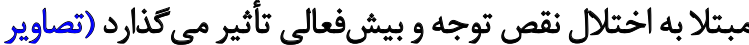

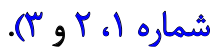
بحث اين مطالعه با هدف بررسى ثأثير بازىدرمانى كروهى بر مشكلات

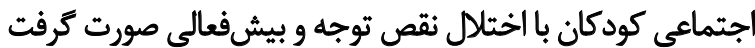

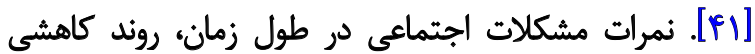

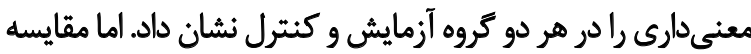

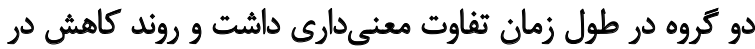

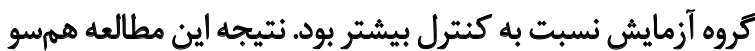

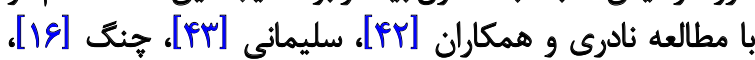

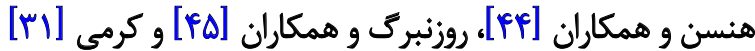

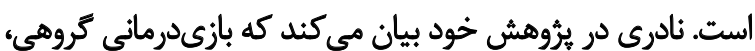




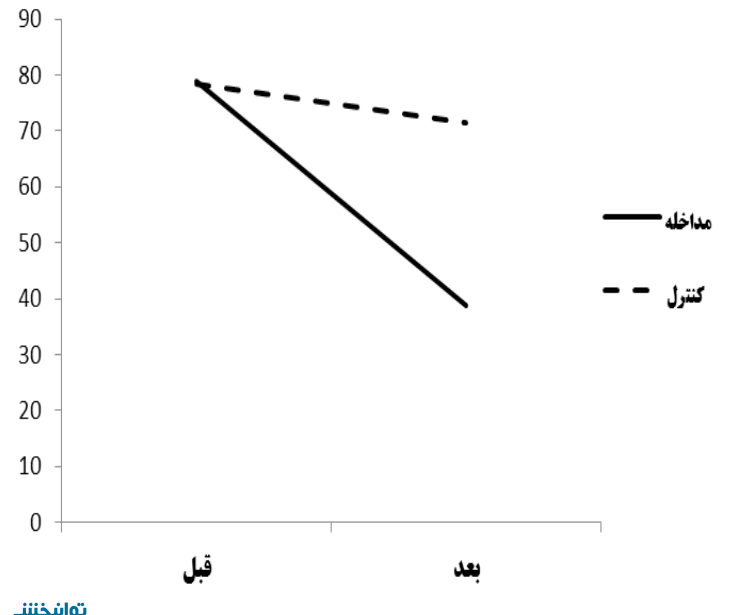

تصوير r. روند تغييرات ميانكين قانونشكنى قبل و بعد از مداخله در دو كروه توانبننى

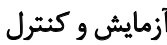

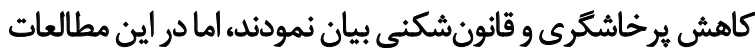

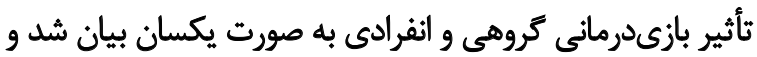

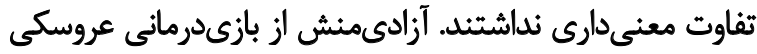

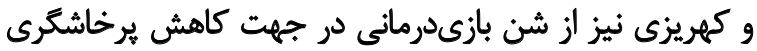

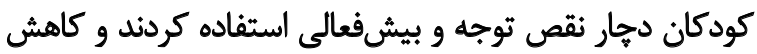

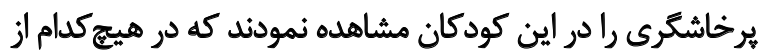

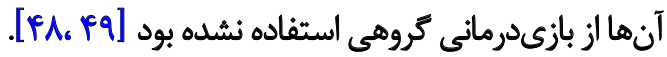
از جنبه مشاهدهاى و كلينيكى، تا قبل از بركزارى جلسات

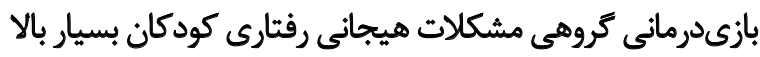

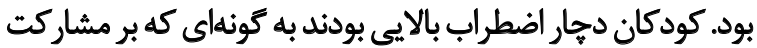

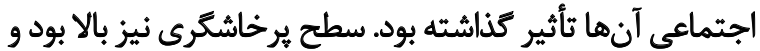

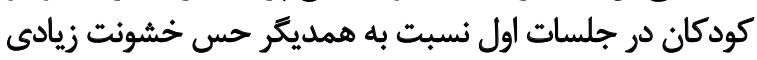

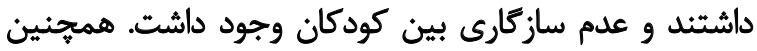

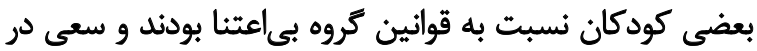

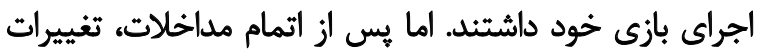

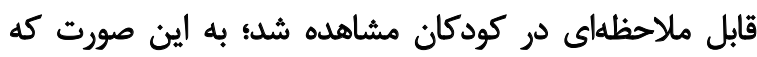

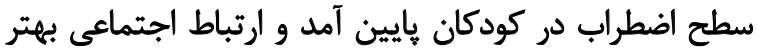

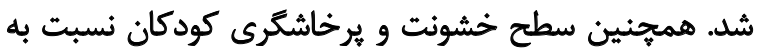

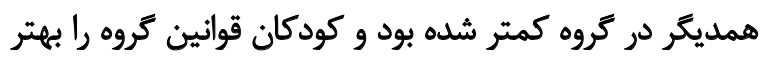
رعايت مي كردند.

والدين نيز يس از مداخلات، بهبود كلى ارتباط كودكان با بارئ

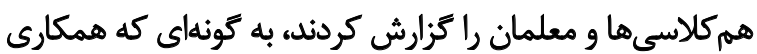

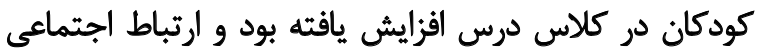

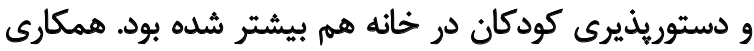

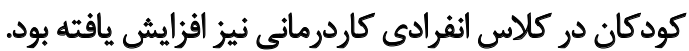

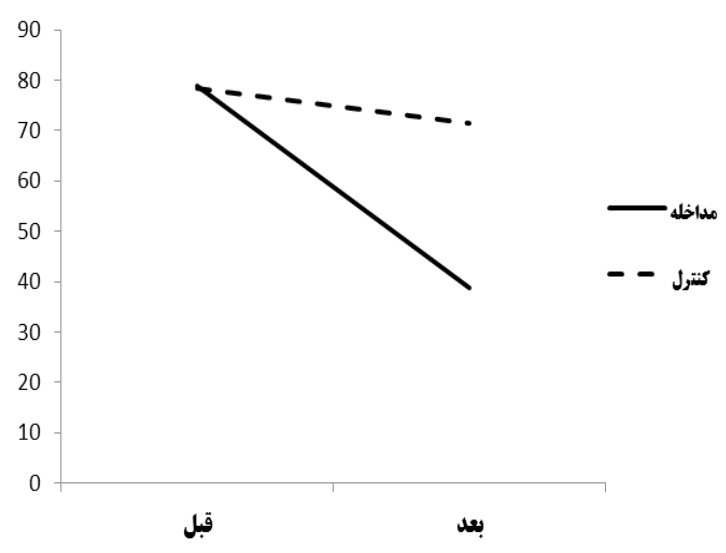

توانبخننى

تصوير ا. روند تغييرات ميانئين مشكلات اجتماعي قبل و بعد از مداخله در

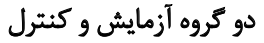

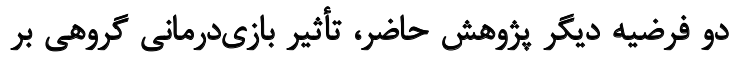

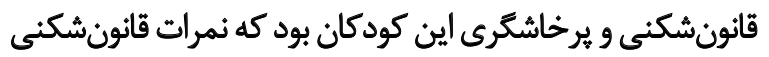

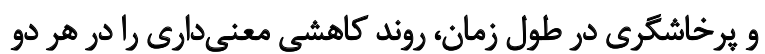

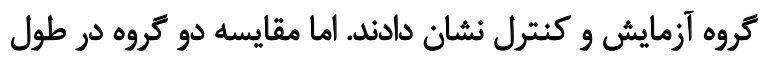

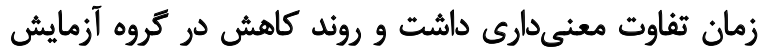

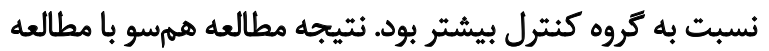

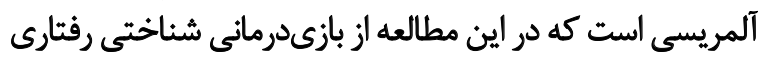

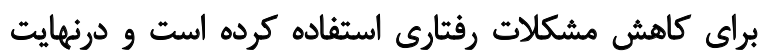

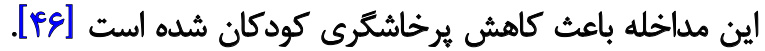

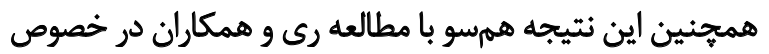

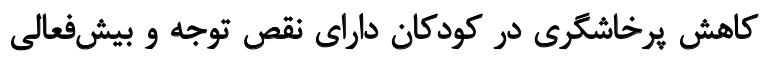

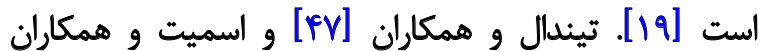

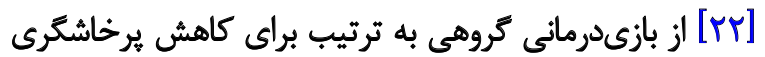

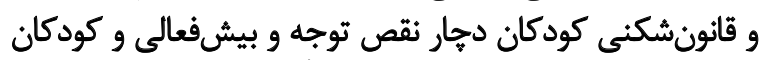

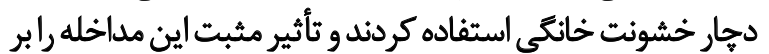

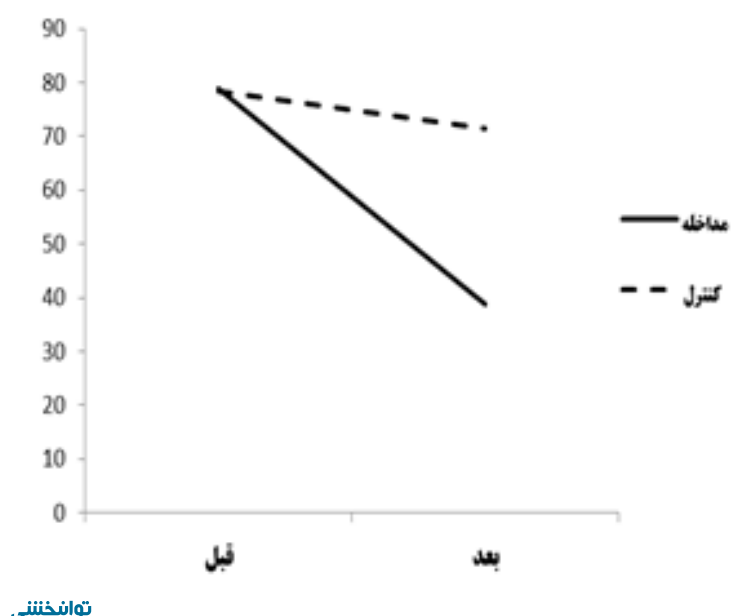

تصوير با. روند تغييرات ميانكين برخاشكرى قبل و بعد از مداخله در دو كروه

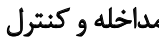




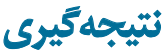

با توجه به مؤثر بودن بازىدرمانى گروهى بر بهبود برديون مشكلات

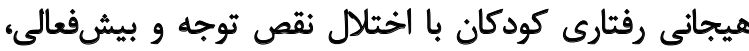

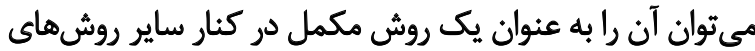

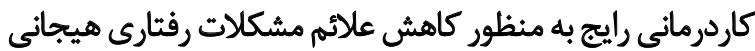

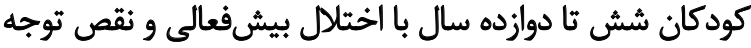
مفيد دانسيث.

محدوديتهاى اصلى يروهش حاضر عبارتند ازئ نمونه كيرى به

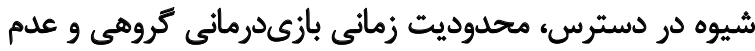
بررسى ماندكارى درمان.

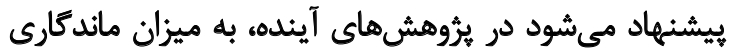

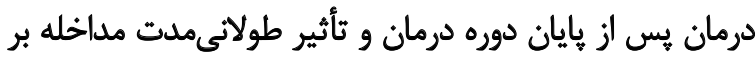
اختلالات رفتارى كودكان با نقص توجه و بيش دان ونعالى توجه شود.

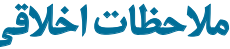

\section{يميروى أز أصول الخاق يُوهش}

اين يزوهش داراى كد اخلاق از كميته اخلاق دانشخاه علوم

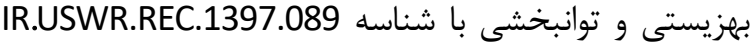

$$
\text { كامي مالى }
$$

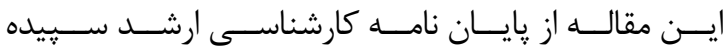

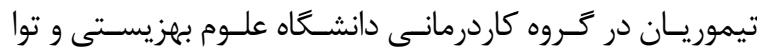

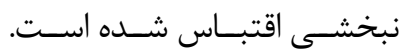

$$
\text { مشاركت نويسندكّان }
$$

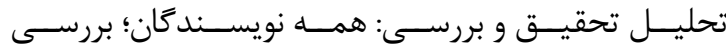

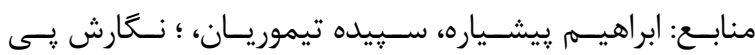

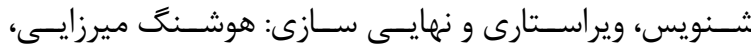

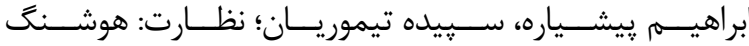

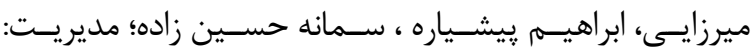

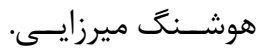

$$
\text { تعارض مثافع }
$$

بنابر اظهار نويسندكان اين مقاله تعارض منافع ندارد.

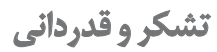

نويسندكان بر خود لازم ميدانند از كارمندان كلينيكهاى كاردرمانى توحيد مراتب تشكر و قدردانى خود را ابراز كنند. 


\section{References}

[1] American Psychiatric Association. Diagnostic and Statistical Manual of Mental Disorders. Washington D.C.: American Psychiatric Association; 2013

[2] Ensafi E, Rostami R, Moghadas Tabrizi Y. Effectiveness of neurofeedback on the event-related potentials in children with attention deficit/hyperactivity disorder. Practice in Clinical Psychology. 2015; 3(2):121-8. http://jpcp.uswr.ac.ir/article-1-252-en.html

[3] Vien TE. Examining the effectiveness of play-based interventions with a focus on executive functioning deficits for children with ADHD [PhD. dissertation]. Chicago, IL: The Chicago School of Professional Psychology; 2015. https://search.proquest.com/openview/fb23aaba9a037aee82d8f34895041130/1

[4] Gau SSF, Lin YJ, Shang CY, Liu SK, Chiu YN, Soong WT. Emotional/behavioral problems and functional impairment in clinicand community-based children with attention-deficit/hyperactivity disorder in Taiwan. Journal of Abnormal Child Psychology. 2010; 38(4):521-32. [DOI:10.1007/s10802-009-9381-6] [PMID]

[5] Salamati A, Hosseini S A, Haghgou H. [Effectiveness of vestibular stimulation on visual attention in children with attention deficit hyperactivity disorder (Persian)]. Archives of Rehabilitation. 2014; 15(3):18-25. http://rehabilitationj.uswr.ac.ir/article-1-1333-en.html

[6] Khoushabi K, Pouretemad H, Mohammadi M, Houman A, Biglarian A, Towfigh N. Prevalence of attention deficit hyperactivity disorder and other coexisting disorder among school students in Tehran. Research Project. Tehran: University of Social Welfare and Rehabilitation Sciences; 2002.

[7] Watson CC. Influence of parental collaboration on group therapy outcomes for anxious children [PhD. dissertation]. Ann Arbor: The Catholic University of America; 2016.

[8] Fazli N, Sajjadian I. [The effectiveness of mindfull parenting education on child behaviors problems and parenting stress among mothers with attention-deficit hyperactivity disorder (Persian)]. Knowledge \& Research in Applied Psychology. 2017; 17(3):26-35. http:// jst-p.khuisf.ac.ir/article_534023.html

[9] Hannesdottir DK, Ingvarsdottir E, Bjornsson A. The OutSMARTers program for children with ADHD: A pilot study on the effects of social skills, self-regulation, and executive function training. Journal of Attention Disorders. 2017; 21(4):353-64. [DOI:10.1177/1087054713520617] [PMID]

[10] Alavinezhad R, Mousavi M, Boojari S. Sleep problems and aggressive behavior in children with ADHD. Practice in Clinical Psychology. 2015; 3(2):107-12. http://jpcp.uswr.ac.ir/article-1-241-en.html

[11] Khademi M, Ayatmehr F, Khosravan Mehr N, Razjooyan K, Davari Ashtiani R, Arabgol F. Evaluation of the effects of positive parenting program on symptoms of preschool children with attention deficit hyperactivity disorder. Practice in Clinical Psychology. 2019; 7(1):11-20. [DOI:10.32598/jpcp.7.1.11]

[12] Biederman J, Spencer T, Lomedico A, Day H, Petty CR, Faraone SV. Deficient emotional self-regulation and pediatric attention deficit hyperactivity disorder: A family risk analysis. Psychological Medicine. 2012; 42(3):639-46. [DOI:10.1017/S0033291711001644] [PMID]
[13] Factor PI, Reyes RA, Rosen PJ. Emotional impulsivity in children with ADHD associated with comorbid--not ADHD--symptomatology. Journal of Psychopathology and Behavioral Assessment. 2014; 36(4):530-41. [DOI:10.1007/s10862-014-9428-z]

[14] Ashori M, Yazdanipour M. [Investigation of the effictiveness of group play therapy training with cognitive-behavioral approach on the social skills of students with intellectual disability (Persian)]. Archives of Rehabilitation. 2018; 19(3):262-75. [DOI:10.32598/ ri.19.3.262]

[15] Jordan J. Developing an integrative play therapy group model for middle school male students to address bullying behaviors $[\mathrm{PhD}$. dissertation]. Clinton, MS: Mississippi College; 2016. https://eric. ed.gov/?id=ED575329

[16] Cheng YJ. Impact of child-centered group play therapy on socialemotional assets of kindergarten children $[\mathrm{PhD}$. dissertation]. Denton, TX: University of North Texas; 2015. https://search.proquest. com/openview/00d1bf61d7dab33d2cb0f3e908dc6f42/1

[17] Pleiss ME. Adventure camp: Evaluation of an experiential learning social skills intervention for children with ADHD and ASD [PhD. dissertation]. Indiana, PA: Indiana University of Pennsylvania; 2016. https://search.proquest.com/openview/be186c85c37ff58b4f1219207313ff52/1

[18] Rafati F, Pourmohamadreza-Tajrishi M, Pishyareh E, Mirzaei H, Biglarian A. Research paper: Effectiveness of group play therapy on the communication of 5-8 years old children with high functioning autism (Persian)]. Archives of Rehabilitation. 2016; 17(3):200-11. [DOI:10.21859/jrehab-1703200]

[19] Ray DC, Schottelkorb A, Tsai MH. Play therapy with children exhibiting symptoms of attention deficit hyperactivity disorder. International Journal of Play Therapy. 2007; 16(2):95-111. [DOI:10.1037/1555-6824.16.2.95]

[20] Parten MB. Social participation among pre-school children. The Journal of Abnormal and Social Psychology. 1932; 27(3):243-69. [DOI:10.1037/h0074524]

[21] Anderson-McNamee JK, Bailey SJ. The importance of play in early childhood development [Internet]. 2010 [Updated 2010 April]. Available from: http://health.msuextension.org/documents/ MT201003HR.pdf

[22] Smith N, Landreth G. Intensive filial therapy with child witnesses of domestic violence: A comparison with individual and sibling group play therapy. International Journal of Play Therapy. 2003; 12(1):6788. [DOI:10.1037/h0088872]

[23] Huth-Bocks A, Schettini A, Shebroe V. Group play therapy for preschoolers exposed to domestic violence. Journal of Child and Adolescent Group Therapy. 2001; 11:19-34 [DOI:10.1023/A:1016693726180]

[24] Mahmoudi Gharaei J, Bina M, Yasami MT, Emami A, Naderi F. [Trauma/grief group play therapy effect on Bam earthquake related emotional and behavioral symptoms in preschool children: A beforeafter trial (Persian)]. Iranian Journal of Pediatrics. 2006; 16(2):13742. https://www.sid.ir/fa/journal/ViewPaper.aspx?ID=46220

[25] Shen YJ. Short-term group play therapy with Chinese earthquake victims: Effects on anxiety, depression and adjustment. International Journal of Play Therapy. 2002; 11(1):43-63. [DOI:10.1037/ h0088856] 
[26] Heshmati R, Onari Asl R, Shokrallah R. [The effectiveness of group play therapy techniques on state anxiety, positive emotions and general compatibility level in special students with learning disabilities (Persian)]. Journal of Learning Disabilities. 2016; 5(4):7-24. http://jld.uma.ac.ir/article_430.html

[27] Baggerly J. The effects of child-centered group play therapy on self-concept, depression, and anxiety of children who are homeless. International Journal of Play Therapy. 2004; 13(2):31-51. [DOI:10.1037/h0088889]

[28] McGuire DE. [Child-centered group play therapy with children experiencing adjustment difficulties (PhD. dissertation)]. Denton, TX: University of North Texas; 2000.

[29] Morshed N, Davoodi I, Babamiri M. [Effectiveness of group play therapy on symptoms of oppositional defiant among children (Persian)]. Journal of Education and Community Health. 2015; 2(3):12-8. [DOI:10.20286/jech-02032]

[30] Jalali S, Kar Ahmadi M, Molavi H, Aghaei A. [The effect of $\operatorname{cog}$ nitive-behavior group play therapy on social phobia in 5-11 years old children (Persian)]. Journal of Research in Behavioural Sciences. 2011; 9(2):104-13. http://rbs.mui.ac.ir/article-1-191-en.html

[31] Karami J, Shafeei B, Heidarisharaf P. [Effectiveness of cognitive-behavioral play therapy on reforming the social maladjustment of female students with intellectual disabilities (Persian)]. Journal of Exceptional Education. 2015; 3(131):21-30. http:// exceptionaleducation.ir/article-1-354-en.html

[32] Dehghan F, Behnia F, Amiri N, Pishyareh E, Safarkhani M. [The effectiveness of using perceptual-motor practices on behavioral disorder among five to eight year old children with attention deficit hyper activity disorder (Persian)]. Advances in Cognitive Sciences. 2010; 12(3):82-96. http://icssjournal.ir/article-1-125-en.html

[33] Kajbaf MB, Oraizi HR, Amiri Sh, Kahnooji R. [The effects of cognitive-behavioral family therapy on children's behavioral problems in divorcing families (Persian)]. Clinical Psychology \& Personality. 2011; 9(1):1-8. http://cpap.shahed.ac.ir/article_2643.html

[34] Mohammad Esmaeel E. [Adaptation and Standardization of Child Symptom Inventory-4 (CSI-4) (Persian)]. Research on Exceptional Childre. 2007; 7(1):79-96. http://joec.ir/article1-388-en.html

[35] Nakamura BJ, Ebesutani C, Bernstein A, Chorpita BF. A psychometric analysis of the child behavior checklist DSM-oriented scales. Journal of Psychopathology and Behavioral Assessment. 2009; 31(3):178-89. [DOI:10.1007/s10862-008-9119-8]

[36] Achenbach TM, Ruffle TM. The child behavior checklist and related forms for assessing behavioral/emotional problems and competencies. Pediatrics in Review. 2000; 21(8):265-71. [DOI:10.1542/pir.21-8-265] [PMID]

[37] Nematollahi M, Tahmasebi S. [The effectiveness of parents' skills training program on reducing children's behavior problems (Persian)]. Journal of Family Research. 2014; 10(2):159-74. http://jfr.sbu.ac.ir/article_96560.html

[38] Minaee A. [Adaptation and standardization of child behavior checklist, youth self-report, and teacher's report forms (Persian)]. Journal of Exceptional Children. 2006; 6(1):529-58. http://joec. ir/article-1-416-en.html
[39] Yazdkhasti F, Oreyzi H. [Standardization of child, parent and teacher's forms of child behavior checklist in the city of Isfahan (Persian)]. Iranian Journal of Psychiatry and Clinical Psychology. 2011; 17(1):60-70. http://ijpcp.iums.ac.ir/article-1-1318-en.html

[40] Ghodousi N, Sajedi F, Mirzaie H, Rezasoltani P. The effectiveness of cognitive-behavioral play therapy on externalizing behavior problems among street and working children. Iranian Rehabilitation Journal. 2017; 15(4):359-66. [DOI:10.29252/nrip. irj.15.4.359]

[41] Teimourian S, Mirzaie H, Pishyare E, Hosseinzade S. [The effectiveness of group play therapy on social problems, aggression, and delinquent of children with attention deficit and hyperactivity disorder in Isfahan 6-12 (Persian)]. Archives of Rehabilitation. 2020; 21(3). http://rehabilitationj.uswr.ac.ir/article-1-2748-en.html

[42] Naderi F, Heidarie A, Bouron L, Asgari P. The efficacy of play therapy on ADHD, anxiety and social maturity in 8 to 12 years aged clientele children of Ahwaz metropolitan counseling clinics. Journal of Applied Sciences. 2010; 10(3):189-95. [DOI:10.3923/ jas.2010.189.195]

[43] Soleimany Adriany S, Sadeghi Hosnije AH, Zerehpoush A, Rabie M, Abedi A, Esmaeeli S. [Effectiveness of rhythmic games on social skills of children with attention deficit hyperactive disorder (Persian)]. Journal of Gorgan University of Medical Sciences. 2016; 18(2):91-6. http://goums.ac.ir/journal/article-1-2767-en.html

[44] Hansen S, Meissler K, Ovens R. Kids together: A group play therapy model for children with ADHD symptomalogy. Journal of Child and Adolescent Group Therapy. 2000; 10(4):191-211. [DOI:10.1023/A:1016631228545]

[45] Rosenberg L, Maeir A, Yochman A, Dahan I, Hirsch I. Effectiveness of a cognitive-functional group intervention among preschoolers with attention deficit hyperactivity disorder: A pilot study. American Journal of Occupational Therapy. 2015; 69(3):6903220040p1-8. [DOI:10.5014/ajot.2015.014795] [PMID]

[46] Almeraisi MJ. Effectiveness of cognitive-behavioral play therapy with children who have symptoms of Attention Deficit Hyperactivity Disorder (ADHD) [PhD. dissertation]. Alhambra, CA: Alliant International University; 2010.

[47] Tyndall-Lind A, Landreth GL, Giordano MA. Intensive group play therapy with child witnesses of domestic violence. International Journal of Play Therapy. 2001; 10(1):53-83. [DOI:10.1037/ h0089443]

[48] Azadimanesh P, hossein khanzadeh A, Hakim-javadi M, Vatankhah M. [Effect of puppet play therapy on aggression of children with ADHD (Persian)]. Studies in Medical Sciences. 2017; 28(2):83-90. http://umj.umsu.ac.ir/article-1-3850-en.html

[49] Kahrizi S, Momeni KM, Moradi A. [The effectiveness of sand play therapy in reducing aggression/ hyperactivity in preschoolers (Persian)]. Counseling Culture and Psycotherapy. 2014; 5(18):111-35. https://qccpc.atu.ac.ir/article_581.html 
This Page Intentionally Left Blank 